\title{
HOLOMORPHIC FUNCTIONAL CALCULUS ON UPPER TRIANGULAR FORMS IN FINITE VON NEUMANN ALGEBRAS
}

\author{
K. DYKEMA*, F. SUKOCHEV ${ }^{\S}$, AND D. ZANIN $§$
}

\begin{abstract}
The decompositions of an element of a finite von Neumann algebra into the sum of a normal operator plus an s.o.t.-quasinilpotent operator, obtained using the Haagerup-Schultz hyperinvariant projections, behave well with respect to holomorphic functional calculus.
\end{abstract}

\section{InTRODUCTION AND DESCRIPTION OF RESUlTS}

This note concerns the decomposition theorem for elements of a finite von Neumann algebra, recently proved in [2]. In that paper, given a von Neumann algebra $\mathcal{M}$ with a normal, faithful, tracial state $\tau$, by using the hyperinvariant subspaces found by Haagerup and Schultz 3 and their behavior with respect to Brown measure, for every element $T \in \mathcal{M}$ we constructed a decomposition $T=N+Q$ where $N \in \mathcal{M}$ is a normal operator whose Brown measure agrees with that of $T$ and where $Q$ is an s.o.t.-quasinilpotent operator. An element $Q \in \mathcal{M}$ is said to be s.o.t.-quasinilpotent if $\left(\left(Q^{*}\right)^{n} Q^{n}\right)^{1 / n}$ converges in the strong operator topology to the zero operator - by Corollary 2.7 in [3], this is equivalent to the Brown measure of $Q$ being concentrated at 0 . In fact, $N$ is obtained as the conditional expectation of $T$ onto the (abelian) subalgebra generated by an increasing family of Haagerup-Schultz projections.

The Brown measure [1] of an element $T$ of a finite von Neumann algebra is a sort of spectral distribution measure, whose support is contained in the spectrum $\sigma(T)$ of $T$. We will use $\nu_{T}$ to denote the Brown measure of $T$. The Brown measure behaves well under holomorphic (or Riesz) functional calculus. Indeed, Brown proved (Theorem 4.1 of [1]) that if $h$ is holomorphic on a neighborhood of the spectrum of $T$, then $\nu_{h(T)}=\nu_{T} \circ h^{-1}$ (the push-forward measure by the function $h$ ).

In this note, we prove the following:

Theorem 1. Let $T$ be an element of a finite von Neumann algebra $\mathcal{M}$ (with fixed normal, faithful tracial state $\tau$ ) and let $T=N+Q$ be a decomposition from [2], with $N$ normal, $\nu_{N}=\nu_{T}$ and $Q$ s.o.t.-quasinilpotent.

(i) Let $h$ be a complex-valued function that is holomorphic on a neighborhood of the spectrum of $T$. Then

$$
h(T)=h(N)+Q_{h},
$$

where $Q_{h}$ is s.o.t.-quasinilpotent.

2000 Mathematics Subject Classification. 47C15.

* Research supported in part by NSF grant DMS-1202660.

$\S$ Research supported by ARC. 
(ii) If $0 \notin \operatorname{supp} \nu_{T}$ (so that $N$ is invertible), then

$$
T=N\left(I+N^{-1} Q\right)
$$

and $N^{-1} Q$ is s.o.t.-quasinilpotent.

The key result for the proof is Lemma 22 of [2], which allows us to reduce to the case when $N$ and $Q$ commute. Before using this, we require a few easy results about s.o.t.-quasinilpotent operators on Hilbert space.

Lemma 2. Let $\mathfrak{A}$ be a unital algebra and let $N, Q \in \mathfrak{A}, T=N+Q$ and suppose that both $N$ and $T$ are invertible. Then

$$
T^{-1}=N^{-1}-T^{-1} Q N^{-1} \text {. }
$$

Proof. We have

$$
T^{-1}-N^{-1}=T^{-1}(N-T) N^{-1}=-T^{-1} Q N^{-1} .
$$

Lemma 3. Let $A$ and $Q$ be bounded operators on a Hilbert space $\mathcal{H}$ such that $A Q=$ $Q A$ and suppose $Q$ is s.o.t.-quasinilpotent. Then $A Q$ is s.o.t.-quasinilpotent.

Proof. We have $(A Q)^{n}=A^{n} Q^{n}$ and

$$
\left((A Q)^{*}\right)^{n}(A Q)^{n}=\left(Q^{*}\right)^{n}\left(A^{*}\right)^{n} A^{n} Q^{n} \leq\|A\|^{2 n}\left(Q^{*}\right)^{n} Q^{n} .
$$

By Loewner's Theorem, for $n \geq 2$ the function $t \mapsto t^{2 / n}$ is operator monotone and we have

$$
\left(\left((A Q)^{*}\right)^{n}(A Q)^{n}\right)^{2 / n} \leq\|A\|^{4}\left(\left(Q^{*}\right)^{n} Q^{n}\right)^{2 / n}
$$

Thus, for $\xi \in \mathcal{H}$, we have

$$
\begin{aligned}
\left\|\left(\left((A Q)^{*}\right)^{n}(A Q)^{n}\right)^{1 / n} \xi\right\|^{2}= & \left\langle\left(\left((A Q)^{*}\right)^{n}(A Q)^{n}\right)^{2 / n} \xi, \xi\right\rangle \\
& \leq\|A\|^{4}\left\langle\left(\left(Q^{*}\right)^{n} Q^{n}\right)^{2 / n} \xi, \xi\right\rangle=\|A\|^{4}\left\|\left(\left(Q^{*}\right)^{n} Q^{n}\right)^{1 / n} \xi\right\|^{2} .
\end{aligned}
$$

Since $Q$ is s.o.t.-quasinilpotent, this tends to zero as $n \rightarrow \infty$.

Proposition 4. Let $N$ and $Q$ be bounded operators on a Hilbert space and suppose $N Q=Q N$ and $Q$ is s.o.t.-quasinilpotent. Let $T=N+Q$. Let $h$ be a function that is holomorphic on a neighborhood of the union $\sigma(T) \cup \sigma(N)$ of the spectra of $T$ and $N$. Then $h(T)$ and $h(N)$ commute, and $h(T)-h(N)$ is s.o.t.-quasinilpotent.

Proof. . If $\lambda$ is outside of $\sigma(T) \cup \sigma(N)$, then by Lemma 2 ,

$$
(T-\lambda)^{-1}=(N-\lambda)^{-1}-(T-\lambda)^{-1} Q(N-\lambda)^{-1} .
$$

Let $C$ be a contour in the domain of the complement $\sigma(T) \cup \sigma(N)$, with winding number 1 around each point in $\sigma(T) \cup \sigma(N)$. Then

$$
\begin{aligned}
h(T) & =\frac{1}{2 \pi i} \int_{C} h(\lambda)(\lambda-T)^{-1} d \lambda \\
h(N) & =\frac{1}{2 \pi i} \int_{C} h(\lambda)(\lambda-N)^{-1} d \lambda .
\end{aligned}
$$


For any complex numbers $\lambda_{1}$ and $\lambda_{2}$ outside of $\sigma(T) \cup \sigma(N)$, the operators $\left(\lambda_{1}-T\right)^{-1}$, $\left(\lambda_{2}-N\right)^{-1}$ and $Q$ commute; thus, $h(T)$ and $h(N)$ commute with each other. Using (1), we have

$$
h(T)-h(N)=\frac{1}{2 \pi i} \int_{C} h(\lambda)(\lambda-T)^{-1} Q(\lambda-N)^{-1} d \lambda=A Q,
$$

where

$$
A=\frac{1}{2 \pi i} \int_{C} h(\lambda)(\lambda-T)^{-1}(\lambda-N)^{-1} d \lambda .
$$

We have $A Q=Q A$. By Lemma 3, $A Q$ is s.o.t.-quasinilpotent.

For the remainder of this note, $\mathcal{M}$ will be a finite von Neumann algebra with specified normal, faithful, tracial state $\tau$.

Lemma 5. Let $T \in \mathcal{M}$. Suppose $p \in \mathcal{M}$ is a $T$-invariant projection with $p \notin\{0,1\}$.

(i) If $T$ is invertible, then $p$ is $T^{-1}$-invariant. Moreover, we have

$$
\begin{aligned}
T^{-1} p & =(p T p)^{-1}, \\
(1-p) T^{-1} & =((1-p) T(1-p))^{-1},
\end{aligned}
$$

where the inverses on the right-hand-sides are in $p \mathcal{M} p$ and $(1-p) \mathcal{M}(1-p)$, respectively.

(ii) The union of the spectra of $p T p$ and $(1-p) T(1-p)$ (in $p \mathcal{M} p$ and $(1-p) \mathcal{M}(1-p)$, respectively) equals the spectrum of $T$.

(iii) If $h$ is a function that is holomorphic on a neighborhood of $\sigma(T)$, then $p$ is $h(T)$-invariant. Moreover, $h(T) p=h(p T p)$.

Proof. For (i), a key fact is that one-sided invertible elements of $\mathcal{M}$ are always invertible. Thus, writing $T=\left(\begin{array}{ll}a & b \\ 0 & c\end{array}\right)$ with respect to the projections $p$ and $(1-p)$ (so that $a=p T p, b=p T(1-p)$ and $c=(1-p) T(1-p))$ writing $T^{-1}=\left(\begin{array}{ll}x & y \\ w & z\end{array}\right)$ and multiplying, we easily see that $a$ and $c$ must be invertible and

$$
T^{-1}=\left(\begin{array}{cc}
a^{-1} & -a^{-1} b c^{-1} \\
0 & c^{-1}
\end{array}\right) .
$$

Thus, $p$ is $T^{-1}$-invariant.

For (ii) we use (i) and the fact that the formula (2) shows that $T$ is invertible whenever $p T p$ and $(1-p) T(1-p)$ are invertible.

For (iii), writing

$$
h(T)=\frac{1}{2 \pi i} \int_{C} h(\lambda)(\lambda-T)^{-1} d \lambda
$$

for a suitable contour $C$, where this is a Riemann integral that converges in norm, the result follows by applying part (i).

For a von Neumann subalgebra $\mathcal{D}$ of $\mathcal{M}$, let $\operatorname{Exp}_{\mathcal{D}}$ and $\operatorname{Exp}_{\mathcal{D}^{\prime}}$, respectively denote the $\tau$-preserving conditional expectations onto $\mathcal{D}$ and, respectively, the relative commutant of $\mathcal{D}$ in $\mathcal{M}$.

Lemma 6. Let $T \in \mathcal{M}$. 
(i) Suppose $0=p_{0} \leq p_{1} \leq \cdots \leq p_{n}=1$ are $T$-invariant projections and let $\mathcal{D}=\operatorname{span}\left\{p_{1}, \ldots, p_{n}\right\}$. Then the spectra of $T$ and of $\operatorname{Exp}_{\mathcal{D}^{\prime}}(T)$ agree. If $T$ is invertible, then $\operatorname{Exp}_{\mathcal{D}^{\prime}}\left(T^{-1}\right)=\operatorname{Exp}_{\mathcal{D}^{\prime}}(T)^{-1}$.

(ii) Suppose $\left(p_{t}\right)_{0 \leq t \leq 1}$ is an increasing family of T-invariant projections in $\mathcal{M}$ with $p_{0}=0$ and $p_{1}=1$, that is right-continuous with respect to strong operator topology. Let $\mathcal{D}$ be the von Neumann algebra generated by the set of all $p_{t}$. If $T$ is invertible, then so is $\operatorname{Exp}_{\mathcal{D}^{\prime}}(T)$ and $\operatorname{Exp}_{\mathcal{D}^{\prime}}\left(T^{-1}\right)=\operatorname{Exp}_{\mathcal{D}^{\prime}}(T)^{-1}$.

Proof. For (i), we have

$$
\operatorname{Exp}_{\mathcal{D}^{\prime}}(T)=\sum_{j=1}^{n}\left(p_{j}-p_{j-1}\right) T\left(p_{j}-p_{j-1}\right) .
$$

The assertions now follow from repeated application of Lemma 5.

For (ii), using the right-continuity of $p_{t}$ it is easy to choose an increasing family of finite dimensional subalgebras $\mathcal{D}_{n}$ of $\mathcal{D}$ whose union is strong operator topology dense in $\mathcal{D}$. Then $\operatorname{Exp}_{\mathcal{D}_{n}^{\prime}}(T)$ and $\operatorname{Exp}_{\mathcal{D}_{n}^{\prime}}\left(T^{-1}\right)$ converge in strong operator topology to $\operatorname{Exp}_{\mathcal{D}^{\prime}}(T)$ and $\operatorname{Exp}_{\mathcal{D}^{\prime}}\left(T^{-1}\right)$, respectively, and both sequences are bounded. From (i) we have the equality

$$
\operatorname{Exp}_{\mathcal{D}_{n}^{\prime}}(T) \operatorname{Exp}_{\mathcal{D}_{n}^{\prime}}\left(T^{-1}\right)=I
$$

and taking the limit as $n \rightarrow \infty$ yields the desired result.

Lemma 7. Let $T \in \mathcal{M}$ and let $p_{t}$ and $\mathcal{D}$ be as in either part (i) or part (ii) of Lemma 6. Suppose a function $h$ is holomorphic on a neighborhood of the spectrum of T. Then $\operatorname{Exp}_{\mathcal{D}^{\prime}}(h(T))=h\left(\operatorname{Exp}_{\mathcal{D}^{\prime}}(T)\right)$.

Proof. Using that the Riemann integral (3) converges in norm, that $\operatorname{Exp}_{\mathcal{D}^{\prime}}$ is norm continuous and applying Lemma 6, we get

$$
\begin{aligned}
& \operatorname{Exp}_{\mathcal{D}^{\prime}}(h(T))=\frac{1}{2 \pi i} \int_{C} h(\lambda) \operatorname{Exp}_{\mathcal{D}^{\prime}}\left((\lambda-T)^{-1}\right) d \lambda \\
&=\frac{1}{2 \pi i} \int_{C} h(\lambda)\left(\lambda-\operatorname{Exp}_{\mathcal{D}^{\prime}}(T)\right)^{-1} d \lambda=h\left(\operatorname{Exp}_{\mathcal{D}^{\prime}}(T)\right) .
\end{aligned}
$$

For convenience, here is the statement of Lemma 22 of [2] and an immediate consequence.

Lemma 8. Let $T \in \mathcal{M}$. For any increasing, right-continuous family of $T$-invariant projections $\left(q_{t}\right)_{0 \leq t \leq 1}$ with $q_{0}=0$ and $q_{1}=1$, letting $\mathcal{D}$ be the von Neumann algebra generated by the set of all the $q_{t}$, the Fuglede-Kadison determinants of $T$ and $\operatorname{Exp}_{\mathcal{D}^{\prime}}(T)$ agree. Since the same is true for $T-\lambda$ and $\operatorname{Exp}_{\mathcal{D}^{\prime}}(T)-\lambda$ for all complex numbers $\lambda$, we have that the Brown measures of $T$ and $\operatorname{Exp}_{\mathcal{D}^{\prime}}(T)$ agree.

Now we have all the ingredients to prove our main result.

Proof of Theorem 1. In Theorem 6 of [2] the decomposition $T=N+Q$ is constructed by considering an increasing, right-continuous family $\left(p_{t}\right)_{0 \leq t \leq 1}$ of Haagerup-Schultz projections, with $p_{0}=0$ and $p_{1}=1$, that are $T$-invariant, letting $\mathcal{D}$ be the von 
Neumann algebra generated by the set of projections in this family and taking $N=$ $\operatorname{Exp}_{\mathcal{D}}(T)$. In particular, each $p_{t}$ is also $Q$-invariant.

For (i), we need to show that the Brown measure of $h(T)-h(N)$ is the Dirac mass at 0 . By Lemma 5 (iii), each $p_{t}$ is $h(T)$-invariant. So by Lemma 8 , the Brown measures of $h(T)-h(N)$ and $\operatorname{Exp}_{\mathcal{D}^{\prime}}(h(T)-h(N))$ agree. Since $h(N) \in \mathcal{D}$, we have $\operatorname{Exp}_{\mathcal{D}^{\prime}}(h(N))=h(N)$ and by Lemma 7 , we have $\operatorname{Exp}_{\mathcal{D}^{\prime}}(h(T))=h\left(\operatorname{Exp}_{\mathcal{D}^{\prime}}(T)\right)$. Combining these facts we get

$$
\nu_{h(T)-h(N)}=\nu_{h\left(\operatorname{Exp}_{\mathcal{D}^{\prime}}(T)\right)-h(N)} .
$$

We have

$$
\operatorname{Exp}_{\mathcal{D}^{\prime}}(T)=N+\operatorname{Exp}_{\mathcal{D}^{\prime}}(Q)
$$

and $\operatorname{Exp}_{\mathcal{D}^{\prime}}(Q)$ is s.o.t.-quasinilpotent. This last statement follows formally from Lemma 8 and the fact that $Q$ is s.o.t.-quasinilpotent. However, we should mention that the fact that $\operatorname{Exp}_{\mathcal{D}^{\prime}}(Q)$ is s.o.t.-quasinilpotent was actually proved directly in [2] as a step in the proof that $Q$ is s.o.t.-quasinilpotent. In any case, since $N$ and $\operatorname{Exp}_{\mathcal{D}^{\prime}}(T)$ commute and $\operatorname{Exp}_{\mathcal{D}^{\prime}}(Q)$ is s.o.t.-quasinilpotent, by Proposition 4 it follows that $h\left(\operatorname{Exp}_{\mathcal{D}^{\prime}}(T)\right)-h(N)$ is s.o.t.-quasinilpotent. Using (4), we get that $h(T)-h(N)$ is s.o.t.-quasinilpotent, as desired.

For (ii), the projections $p_{t}$ form a right-continuous family, each of which is invariant under $N^{-1} Q$. By Lemma 8 , the Brown measure of $N^{-1} Q$ equals the Brown measure of

$$
\operatorname{Exp}_{\mathcal{D}^{\prime}}\left(N^{-1} Q\right)=N^{-1} \operatorname{Exp}_{\mathcal{D}^{\prime}}(Q)
$$

But since $N^{-1}$ and $\operatorname{Exp}_{\mathcal{D}^{\prime}}(Q)$ commute and since the latter is s.o.t.-quasinilpotent, by Lemma 3, their product (5) is s.o.t.-quasinilpotent.

\section{REFERENCES}

[1] L. G. Brown, Lidskii's theorem in the type II case, Geometric methods in operator algebras (Kyoto, 1983), Pitman Res. Notes Math. Ser., vol. 123, Longman Sci. Tech., Harlow, 1986, pp. 1-35.

[2] K. Dykema, F. Sukochev, and D. Zanin, A decomposition theorem in II $I_{1}$-factors, J. reine angew. Math., to appear, available at http://arxiv.org/abs/1302.1114.

[3] U. Haagerup and H. Schultz, Invariant subspaces for operators in a general II $I_{1}$ factor, Publ. Math. Inst. Hautes Études Sci. 109 (2009), 19-111.

Department of Mathematics, Texas A\&M University, College Station, TX, USA. E-mail address: ken.dykema@math.tamu.edu

School of Mathematics and Statistics, University of new South Wales, KensingTON, NSW, Australia.

E-mail address: f.sukochev@math.unsw.edu.au

School of Mathematics and Statistics, University of new South Wales, KensingTON, NSW, Australia.

E-mail address: d.zanin@math.unsw.edu.au 